\title{
Analysis of Directional Reflectance and Surface Orientation Using Fresnel Theory
}

\author{
Gary A. Atkinson and Edwin R. Hancock \\ Department of Computer Science, \\ University of York, York, YO10 5DD, UK \\ \{atkinson, erh\}@cs.york.ac.uk
}

\begin{abstract}
Polarization of light caused by reflection from dielectric surfaces has been widely studied in computer vision. This paper presents an analysis of the accuracy of a technique that has been developed to acquire surface orientation from the polarization state of diffusely reflected light. This method employs a digital camera and a rotating linear polarizer. The paper also explores the possibility of linking polarization vision with shading information by means of a computationally efficient BRDF estimation algorithm.
\end{abstract}

\section{Introduction}

Many attempts have been made by the computer vision community to exploit the phenomenon of the partial polarization of light caused by reflection from smooth surfaces. Existing work has demonstrated the usefulness of polarization in surface height recovery $[7|6| 8 \mid 1$; overcoming the surface orientation ambiguity associated with photometric stereo [34]; image segmentation [11; recognition and separation of reflection components 1011]; and distinguishing true laser stripes from inter-reflections for triangulation based laser scanning [2]. Polarization vision has been studied for both metallic and dielectric surfaces and both specular and diffuse reflection. However, little work has been carried out that assesses the accuracy of these techniques or to couple these methods with shape from shading or other intensity-based methods.

This paper is concerned with what is probably the most studied of the above applications: shape recovery. In particular, we focus on shape recovery from diffuse reflection from dielectric surfaces since this is the most commonly occurring situation. The paper uses a technique to recover surface normals from polarization that involves a linear polarizer being mounted on a digital camera and images taken as the transmission axis of the polarizer is rotated. We apply this method to objects made from a variety of materials of known shape. The surface orientation prediction based on polarization is then compared to the exact values calculated from the known geometry. The analysis reveals several unstudied features of surface polarization that help to demonstrate where current techniques of polarization vision are adequate and where its use is inappropriate or where more detailed models are required. 
We also use polarization to estimate the "slice" of the bidirectional reflectance distribution function (BRDF) corresponding to the case where the camera and light source are coincident. We do this for objects of unknown shape and compare the results to objects of the same material but known shape. This is of interest for three reasons. Firstly, it complements the accuracy analysis since, again, exact values can be deduced from the known shapes. Secondly, BRDF data may be useful for the shape recovery of surface regions that cause difficulty for polarization vision such as inter-reflections [1]. Finally, BRDF data can be used for realistic image rendering.

\section{Polarization and Reflection}

The Fresnel equations give the ratios of the reflected wave amplitude to the incident wave amplitude for incident light that is linearly polarized perpendicular to, or parallel to, the plane of specular incidence. These ratios depend upon the angle of incidence and the refractive index, $n$, of the reflecting medium. Since the incident light can always be resolved into two perpendicular components, the Fresnel equations are applicable to all incident polarization states. Indeed, throughout this work, we assume that the incident light is unpolarized.

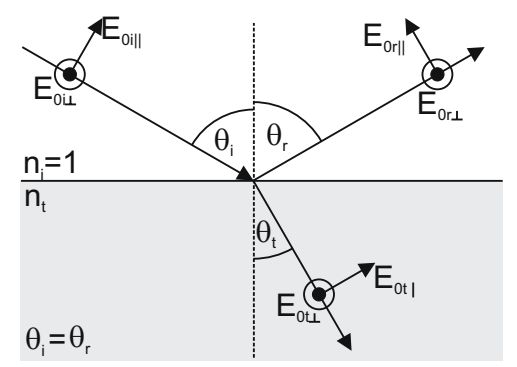

Fig. 1. Definitions. Directions of electric fields are indicated.

For the geometry of Fig. 1, the Fresnel reflection coefficients are [5]

$$
\begin{gathered}
r_{\perp}\left(n_{i}, n_{t}, \theta_{i}\right) \equiv \frac{E_{0 r \perp}}{E_{0 i \perp}}=\frac{n_{i} \cos \theta_{i}-n_{t} \cos \theta_{t}}{n_{i} \cos \theta_{i}+n_{t} \cos \theta_{t}} \\
r_{\|}\left(n_{i}, n_{t}, \theta_{i}\right) \equiv \frac{E_{0 r \|}}{E_{0 i \|}}=\frac{n_{t} \cos \theta_{i}-n_{i} \cos \theta_{t}}{n_{t} \cos \theta_{i}+n_{i} \cos \theta_{t}}
\end{gathered}
$$

where (1) gives the reflection ratio for light polarized perpendicular to the plane of incidence and (2) is for light polarized parallel to the plane of incidence. The angle $\theta_{t}$ can be obtained from the well-known Snell's Law: $n_{i} \sin \theta_{i}=n_{t} \sin \theta_{t}$. Cameras do not measure the amplitude of a wave but the square of the amplitude, or intensity. With this in mind, it is possible to show that the intensity 
coefficients, which relate the reflected power to the incident power, are $R_{\perp}=r_{\perp}^{2}$ and $R_{\|}=r_{\|}^{2}[5]$.

Figure 2 shows the Fresnel intensity coefficients for a typical dielectric as a function of the angle of the incident light. Both reflection and transmission coefficients are shown, where the latter refers to the ratio of transmitted to incident power (the transmission coefficients are simply $T_{\perp}=1-R_{\perp}$ and $T_{\|}=$ $\left.1-R_{\|}\right)$.

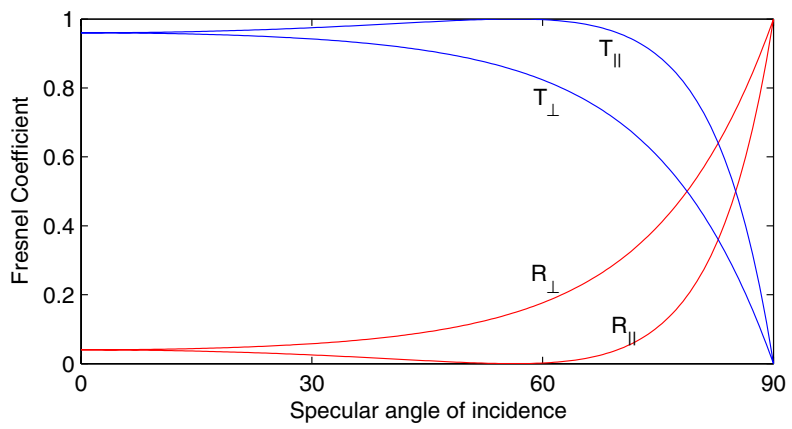

Fig. 2. Reflection and transmission coefficients for a dielectric $(n=1.5)$

The work reported here relies on taking a succession of images of objects with a polarizer mounted on the camera at different angles. As the polarizer is rotated, the measured pixel brightness at a given point varies sinusoidally. Let $I_{\max }$ and $I_{\min }$ be the maximum and minimum intensities in this sinusoid respectively. The degree of polarization is defined to be

$$
\rho=\frac{I_{\max }-I_{\min }}{I_{\max }+I_{\min }}
$$

Careful consideration of Fig. 2 and the Fresnel equations leads to an expression for the degree of polarization in terms of the refractive index and the zenith angle, that is, the angle between the surface normal and the viewing direction. Unfortunately, this equation is only applicable to specular reflection since the process that causes diffuse polarization, the sole concern of this paper, is different, as explained below.

Diffuse polarization is a result of the following process 11]: A portion of the incident light penetrates the surface and is scattered internally. Due to the random nature of internal scattering, the light becomes depolarized. Some of the light is then refracted back into the air, being partially polarized in the process. Snell's Law and the Fresnel equations can be used to predict the degree of polarization of light emerging from the surface at a given angle. Figure 3 shows the Fresnel coefficients for light being refracted back into air. 


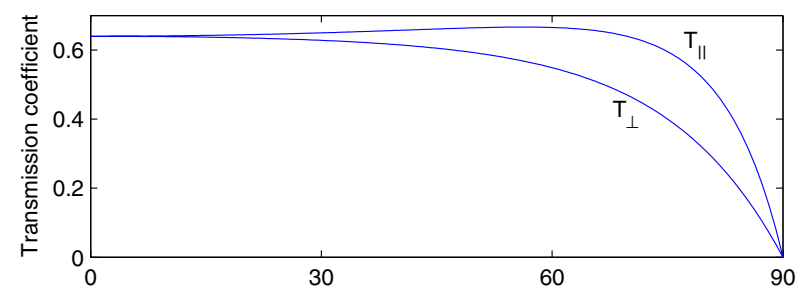

Fig. 3. Fresnel coefficients for light leaving a medium $(n=1.5)$

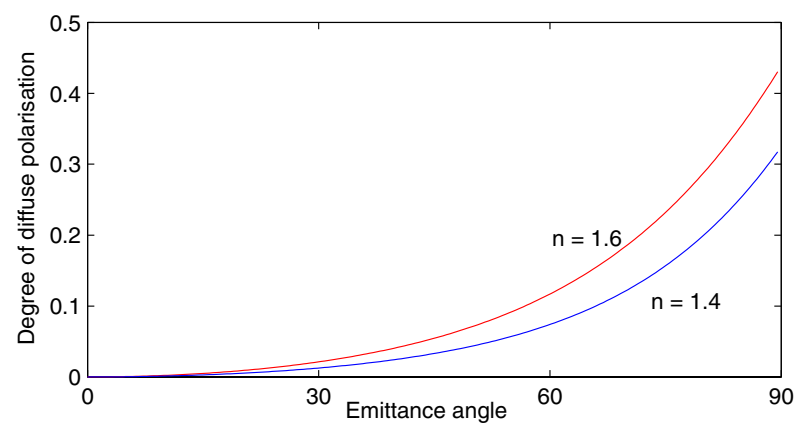

Fig. 4. Degree of polarization for diffuse reflection for two different refractive indices

Using a similar method to that used for specular polarization, an equation for the degree of polarization in terms of the zenith angle and refractive index can be derived:

$$
\rho=\frac{(n-1 / n)^{2} \sin ^{2} \theta}{2-2 n^{2}-(n+1 / n)^{2} \sin ^{2} \theta+4 \cos \theta \sqrt{n^{2}-\sin ^{2} \theta}}
$$

The dependence of the diffuse polarization $\rho$ on the zenith angle $\theta$ is shown in Fig. 4 .

The azimuth angle of the surface normal, i.e. the angle of the projection of the surface normal onto the image plane, is also intimately related to the Fresnel equations. As Fig. 3 shows, diffusely reflected light is reflected most efficiently when polarized parallel to the plane containing the surface normal and the ray reflected towards the camera. The azimuth angle therefore exactly matches the angle of the polarizer that permits greatest transmission.

\section{Polarization Analysis}

Equations (3) and (4) are central to the technique of recovering surface orientation from diffuse polarization. For the experiments described below, the surface normal azimuth and zenith angles were recovered using the following method: For each object, 36 images were taken with a Nikon D70 digital SLR camera, 
with a linear polarizer mounted on the lens, which was rotated by $5^{\circ}$ between successive images. There was just one light source, a small but intense collimated tungsten lamp. The walls, floor and ceiling of the laboratory, as well as the table on which the objects lay, were matte black to avoid inter-reflections from the environment.

As mentioned earlier, pixel brightness varies sinusoidally with polarizer angle. A sinusoid was therefore fitted to the pixel brightnesses for each point on the images. With $I_{\max }$ and $I_{\min }$ taken from this fit, (3) and (4) were used to estimate the zenith angles. The azimuth angle of the surface was taken to match the polarizer angle that allowed greatest transmission.

To assess the accuracy of the method, a set of vertically oriented cylinders of various materials were used. The geometry of a cylinder is convenient for three reasons. First, the analysis can easily be performed for all possible zenith angles. Second, noise can be reduced by taking the average image intensity for each column of pixels. Finally, the structure is simple enough for shape recovery to be performed exactly from a single image. This is simply done by isolating the cylinder from the background and placing semicircles that arch from one side of the object to the other.

Using the method described above, we obtained a set of graphs showing the measured and theoretical zenith angles against position across the cylinder for different materials. Since the azimuth angle of the cylinder is constant, we can also see how the accuracy of azimuth angle estimates vary with zenith angle, if at all. A sample of these results for porcelain, blank photographic paper, photographic paper coated with cling film and normal paper are shown in Fig. 5 The photographic paper is much smoother than normal paper due to its coating. Several other material samples were also analysed, including different paper types, plastics, wax, terracotta and papers coated with inks. The graphs of Fig. 5 provide a good overall representation.

The first point to note about the figures is that, even for normal paper which at the fine scale is very rough, the azimuth angles have been accurately recovered. However, more noise is associated with the rougher surfaces.

There was greater variation in the accuracy of the zenith angle estimates. For Fig. 5, the refractive index used was simply the value that produced greatest similarity between theory and experiment for the material in question. The shiny white porcelain object produced excellent agreement with theory down to very small zenith angles.

The remaining graphs in Fig. [5 demonstrate the complications that can cause the measured zenith angles to deviate from the expected values. The result for blank white photographic paper, for example, is very accurate for large zenith angles but an increasing discrepancy is present as the zenith angle approaches zero. When the paper is coated in cling film, the discrepancy is less marked. Clearly, this suggests that there is a process occurring that is not accounted for by the theory. It is not considered useful to investigate this phenomenon further because the intensity may vary by just a few grey levels in such regions. Therefore, intensity quantization errors prevent extraction of useful data. The 

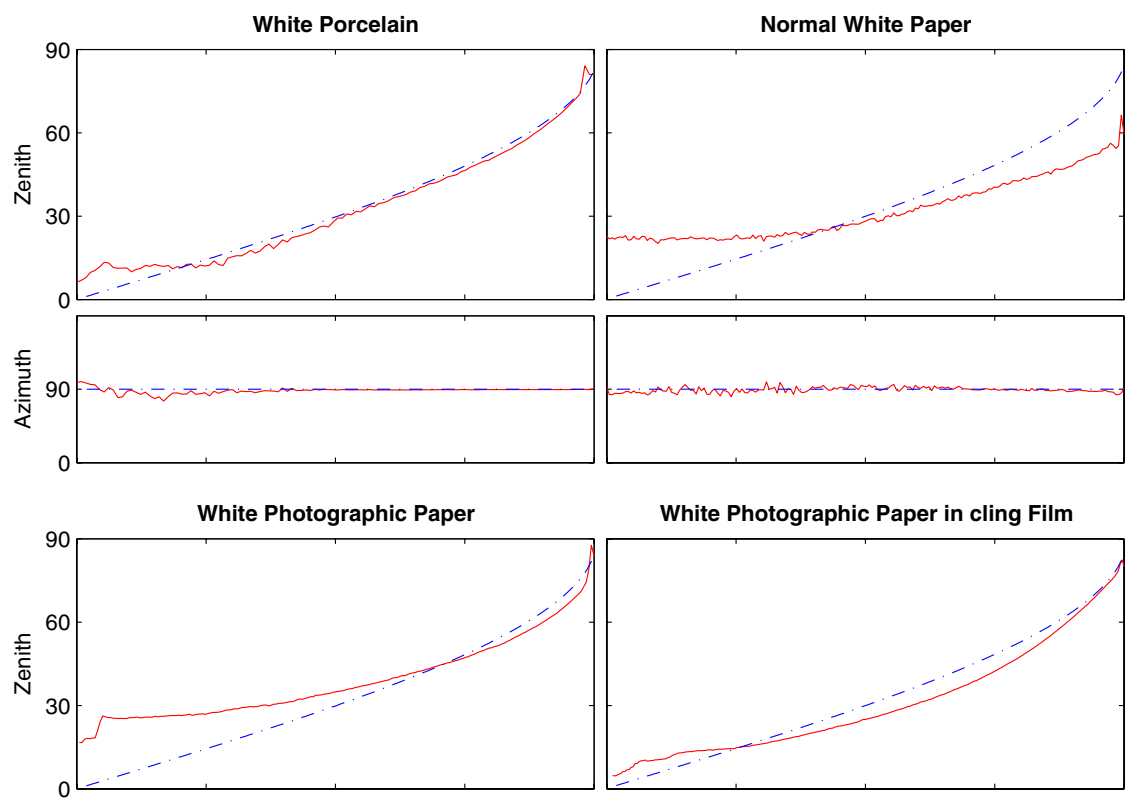

White Photographic Paper in cling Film
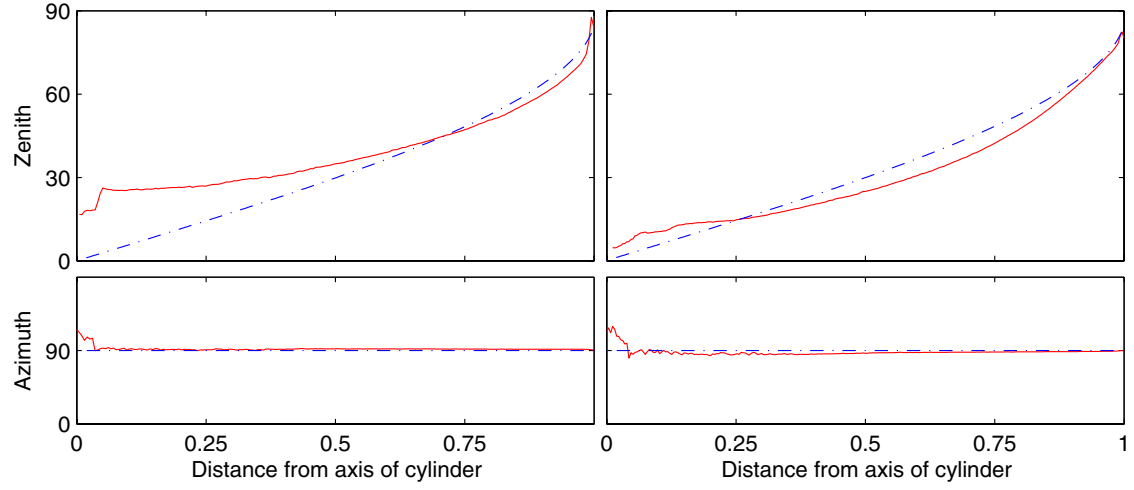

Fig. 5. Plots of measured zenith and azimuth angles (solid lines) across the surfaces of cylinders of different materials. The exact values are indicated by the broken curves.

results for paper, which of course, is a rough matte surface, also show the phenomenon of finite polarization at low zenith angles, as well as depolarizing effects of roughness nearer to the limbs.

\section{Shading Analysis and BRDF Estimation}

We now turn our attention to information contained within the shading of the images. For this analysis normal digital photographs were used (i.e. the polarizer was removed from the camera) although taking the sum of two images with the polarizer angle $90^{\circ}$ different gives the same result (except for an overall intensity reduction due to non-ideal filters). This analysis demonstrates the relative strengths of polarization and shading analysis.

It is not our intention here to present a detailed survey of reflectance models [12] but we are interested in where shading information should be used in place of polarization. First consider the simplest reflectance model: the Lambertian 


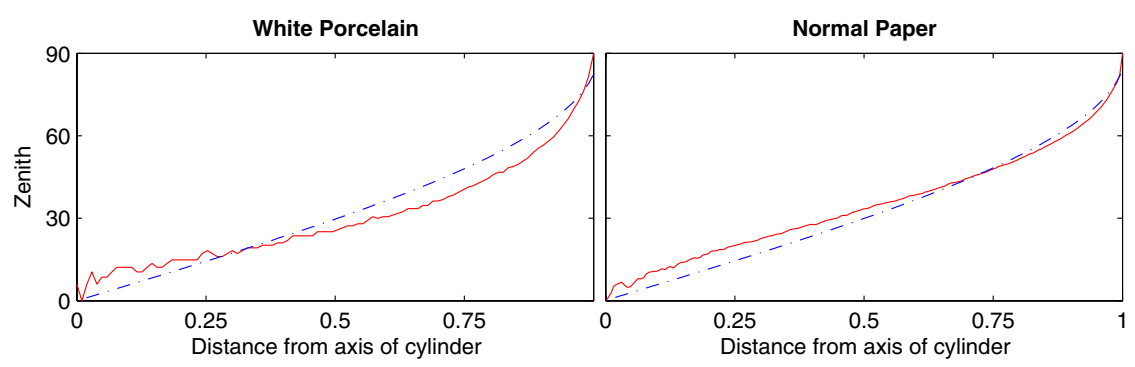

Fig. 6. Estimation of zenith angles using the Lambertian reflectance model (solid lines) compared to ground truth for two materials

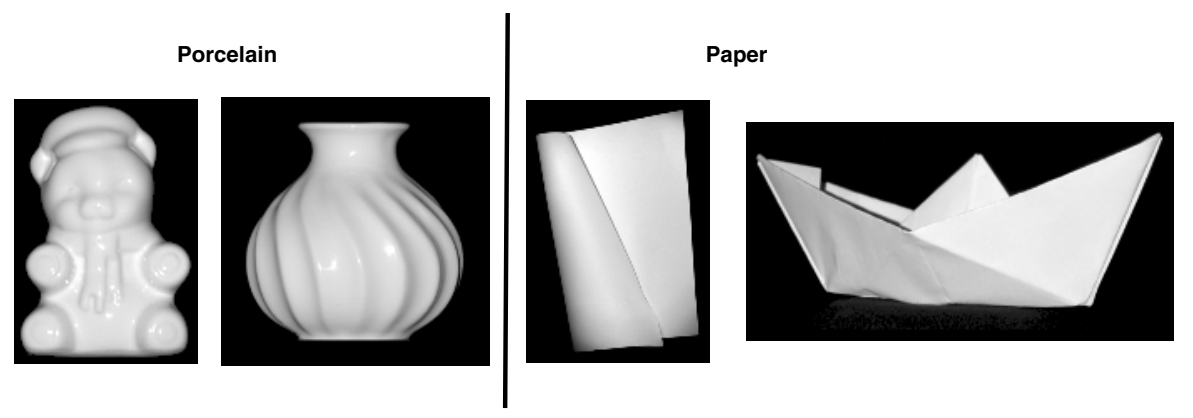

Fig. 7. Some of the objects used in BRDF estimation

approximation. Figure 6 shows the zenith angle prediction using this model for the porcelain and paper samples from Fig. 5. Polarization clearly gave much better results for porcelain, but for paper (a genuinely Lambertian surface), polarization was weaker due to roughness. Note however that the Lambertian model tells us little about the surface azimuth angle, whereas even for paper, this was accurately recovered from polarization up to a $180^{\circ}$ ambiguity caused by the equivalence of phase angles separated by this angle.

For the final contribution of this paper, we consider the BRDF of these two very different materials. In full, the BRDF is the ratio of reflected light to incident light for all possible viewing and illumination directions. Here, we are concerned with estimating the "slice" of the BRDF where the light source and camera are coincident using a single polarization image. The method is very simple and computationally efficient and we compare the results to ground truth and to an intensity based method.

The polarization-based method simply bins the zenith angles recovered from a polarization image (here bin sizes were taken to be $1^{\circ}$ wide) and plots intensity against zenith angle. The intensity based method uses the cumulative distribution of intensity gradients to estimate the zenith angles which then approximates the BRDF in the form of a polar function on a Gauss sphere. Details of this method can be found in [9]. 


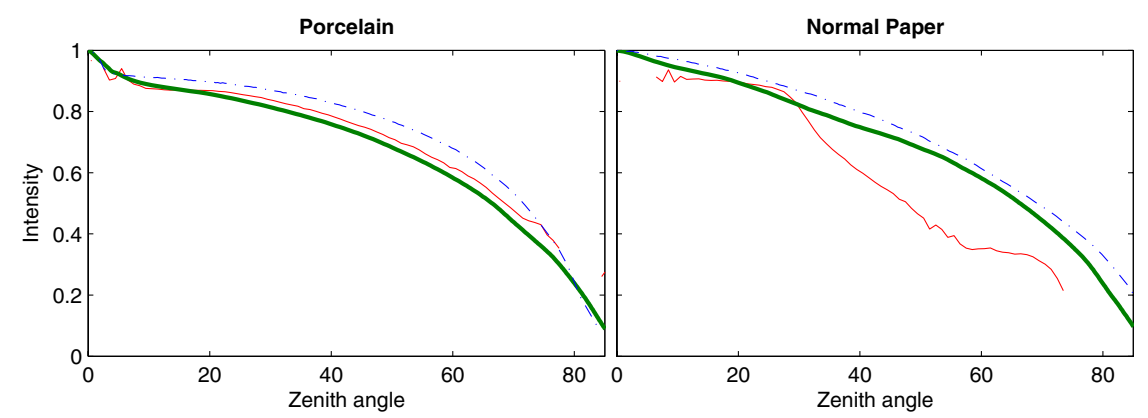

Fig. 8. Estimation of BRDF for porcelain and paper using polarization (thin solid line) and intensity (thick line) compared to the exact curve (broken line). Note the near-perfect overlap between the exact and polarization measurements for large zenith angles for porcelain.

These BRDF estimation methods were applied to objects made of porcelain and paper, some of which are shown in Fig. 7. A BRDF graph was obtained for each object. Figure 8 shows the mean graph for each material. Zenith angles above $85^{\circ}$ are not shown due to the difficulty in obtaining reliable intensity data for these areas. Results are broadly as expected. For porcelain, both methods gave good results with polarization being more accurate. In particular, the polarization method gives almost exact results above about $70^{\circ}$. The random-looking curve for paper shows that BRDF estimation is highly sensitive to surface geometry for that material so intensity-based methods should clearly be used here. The BRDF can be estimated in full by repeating the experiment under many different lighting conditions and interpolating between the positions used to estimate the BRDF under arbitrary illumination conditions.

\section{Conclusion}

This work has presented a sensitivity study of shape from diffuse polarization for various materials. The difference in the accuracy of the method between regions of high and low zenith angles is clearly illustrated by Fig. 5. which also provides a detailed picture of the effects of roughness. Importantly, we see that the surface normal azimuth angles can be accurately determined even for moderately rough surfaces. The BRDF experiments have demonstrated very efficient methods for BRDF estimation from polarization and intensity and has applications in image rendering and combining shape from shading with polarization. The paper clearly identifies strengths and weaknesses of shape from polarization over shape from shading. 


\section{References}

1. G. A. Atkinson and E. R. Hancock. Recovery of Surface Orientation from Diffuse Polarization. To appear: Trans. Image Proc.

2. J. Clark, E. Trucco, and L.B. Wolff. Using light polarization in laser scanning. Image and Vision Computing, 15:107-117, 1997.

3. O. Drbohlav and R. Śára. Unambiguous determination of shape from photometric stereo with unknown light sources. In Proc. of ICCV, pages 581-586, 2001.

4. O. Drbohlav and R. Š́ra. Specularities reduce ambiguity of uncalibrated photometric stereo. In Proc. of ECCV, volume 2, pages 46-62, 2002.

5. E. Hecht. Optics. Addison Wesley Longman, third edition, 1998.

6. D. Miyazaki, M. Kagesawa, and K. Ikeuchi. Transparent surface modelling from a pair of polarization images. IEEE Trans. Patt. Anal. Mach. Intell., 26:73-82, 2004.

7. D. Miyazaki, M. Saito, Y. Sato, and K. Ikeuchi. Determining surface orientations of transparent objects based on polarization degrees in visible and infrared wavelengths. J. Opt. Soc. Am. A, 19:687-694, 2002.

8. S. Rahmann and N. Canterakis. Reconstruction of specular surfaces using polarization imaging. In Proc. CVPR, pages 149-155, 2001.

9. A. Robles-Kelly and E. R. Hancock. Estimating the surface radiance function from single images. To appear: Graphical Models.

10. S. Umeyama. Separation of diffuse and specular components of surface reflection by use of polarization and statistical analysis of images. IEEE Trans. Patt. Anal. Mach. Intell, 26:639-647, 2004.

11. L. B. Wolff and T. E. Boult. Constraining object features using a polarization reflectance model. IEEE Trans. Pattern Anal. Mach. Intell, 13:635-657, 1991.

12. L. B. Wolff, S. K. Nayar, and M. Oren. Improved diffuse reflection models for computer vision. Intl. J. Computer Vision, 30:55-71, 1998. 